Ueber das Verhalten des entfärbten Harns zu Kupferoxyd u. Alkali etc. 127

(Aus dem physiologischen Institut zu Christiania.)

\title{
Ueber das Verhalten des entfärbten Harns zu Kupfer- oxyd und Alkali und den Nachweis von Zucker in demselben.
}

Von

Prof. Worm Finller.

$$
\S 1 \text {. }
$$

\section{Ueber die Entfärbung des Harns.}

Am zweckmässigsten geschieht die Entfärbung des Harns mittelst (wiederholter) Filtration (ohne Erwärmung) durch Thierkohle. So erhält man eine wasserklare Flüssigkeit, welche höchstens Spuren von Harnsäure enthält. Seegen, welcher zuerst diese Methode empfohlen hat, legt besonderen Nachdruck darauf, dass die Thierkohle Blutkohle sein miisste ${ }^{1}$ ); dies muss jedoch als minder wesentlich bezeichnet werden, da nach den hier gewonnenen Erfahrungen Blutkohle und Knochenkohle denselben Nutzen gewähren, vorausgesetzt nur, dass dieselben fein pulverisirt und gut gereinigt sind. Von grösserer Bedeutung ist der Umstand, dass man bei der Filtration genau darauf achtet, dass die sämmtliche Flüssigkeit durch die Kohle passirt, denn hat man gut hierfur gesorgt, sind wiederholte Filtrationen gewöhnlich nicht erforderlich. Um diesen Zweek zu erreichen, bringt man die Kohle auf ein Filter und tropft so lange Harn dazu, bis das Koblenpulver in einen Teig verwandelt ist. In der Mitte des letzteren bildet man nun eine Vertiefung, in welche man nach und nach den Harn einschüttet. Dies muss mit Vorsicht geschehen. Giesst man nämlich

1) Dieses Archiv, Bd. V, 1872, S. 376. 
auf ein Mal zu viel Harn zu, so dass dieser sich über dem Niveau der Kohlenschicht des Filters befindet, so gewährt die Filtration nicht den beabsichtigten Nutzen.

\section{$\S 2$.}

\section{Das Vermögen des entfärbten zuckerfreien Harns Kupferoxyd- hydrat in alkalischer Flüssigkeit aufzulösen.}

Gew öhnlicher zuckerfreier Harn besitzt nach der Entfärbung immer noch das Vermögen, die Ausfällung einer gewissen Menge Kupferoxydhydratinalkalischer Mischung zu verhindern, jedoch in geringerem Grade; cfr. folgende Versuche mit eben demselben Harn vor und nach der Entfärbung.

1. Vor der Entfärbung; spec. Gew. 1,024.

$5 \mathrm{ccm}$ Harn, 2,5 ccm normaler Kalilange ergaben mit 1,25 cem Kupfersulfatlösung $(2,5 \mathrm{pCt}$.) eine klare Flüssigkeit, dagegen nicht mit $1,5 \mathrm{ccm}$ $\mathrm{CuSO}_{4}$. Wurde $\mathrm{CuSO}_{4}$ vor der Kalilauge zugesetzt, war die Flüssigkeit ganz klar grünblau bei Anwendung von $2 \mathrm{ccm} \quad \mathrm{CuSO}_{4}$; dagegen nicht mehr bei $2,5 \mathrm{ccm}$.

2. Nach der Entfärbung; spec. Gew. 1,022.

$5 \mathrm{ccm}$ Harn und 2,5 $\mathrm{cm} \mathrm{KOH} \mathrm{gaben} \mathrm{mit} 0,75 \mathrm{~cm} \mathrm{CuSO}_{4}$ klare Flüssigkeit, dagegen nicht mit $1 \mathrm{ccm}$. Wurde $\mathrm{CuSO}_{4}$ vor dem $\mathrm{KOH}$ zugesetzt, war die Flüssigkeit klar bläulich bei Anwendung von 1,75 ccm, dagegen nicht mehr bei $\left.2 \mathrm{ccm}^{1}\right)$.

Da der entfärbte Harn immer noch (in der Regel) das Vermögen besitzt, eine nicht geringe Menge Kupferoxyd aufzunehmen, so folgt hieraus, dass noch andere Stoffe, ausser den Farbstoffen und der Harnsäure, welche beide bekanntlich von der Kohle zurïckgehalten werden, hierbei in wesentlichem Grade in Betracht kommen.

\section{$\S 3$.}

\section{Das Reductionsvermögen des entfärbten zuckerfreien Harns.}

Durch die Entfärbung muss das Reductionsvermögen des Harns abnehmen, da die Harnsäure von der Kohle zuriekgehalten wird. Dies ist dagegen nicht der Fall mit dem Kreatinin, und, wie es scheint, auch nicht mit der Substanz (resp. den Substanzen),

1) Wie ersichtlich, wird ebenso wie im ursprünglichen Harne mehr Kupferoxyd aufgelöst, wenn man Kupfersulfat vor dem Alkali zusetzt; cfr. S. 88 , Z. 5 v. o., wo statt "im.letzterem Falle" ,im ersteren Falle" zu lesen ist. 
Ueber das Verhalten des entfärbten Harns zu Kupferoxyd u. Alkali etc. 129

welche Wismuthoxyd reduciren. Ich habe früher darauf aufmerksam gemacht, dass viele zuckerfreie Harne im Stande sind, Wismuthoxyd in alkalischer Lösung nach andauerndem Kochen zu reduciren. Dieses Vermögen ist bei den bisher ausgefithrten Versuchen nach der Filtration durch Kohle nicht verschwunden, so dass die resp. Substanzen kaum in "höherem Grade durch letztere zurïckgehalten werden.

Um nun darüber Gewissheit zu erhalten, ob die Kohle auch andere reducirende Substanzen ausser der Harnsäure aufnimmt, hat Herr Dr. H. J. Vetlesen vermittelst der Knapp'schen Flüssigkeit vergleichende Bestimmungen über das Reductionsvermögen ausgefuihrt: a) im ursprünglichen Harn, b) in demselben nach Ausfällung der Harnsäure durch Salzsäure, und c) in dem entfärbten Harn.

\begin{tabular}{|c|c|c|c|c|c|}
\hline \multirow{3}{*}{$\mathrm{Nr}$. } & \multirow{3}{*}{ Spec. Gew. } & \multirow{3}{*}{$\begin{array}{l}\text { Harnsäure- } \\
\text { gehalt. }\end{array}$} & \multicolumn{3}{|c|}{ Reductionsvermögen ${ }^{1}$ ). } \\
\hline & & & \multicolumn{2}{|c|}{ Vor der Entfärbung. } & \multirow{2}{*}{$\begin{array}{l}\text { Nach der } \\
\text { Entfärbung }\end{array}$} \\
\hline & & & $\begin{array}{l}\text { Vor Ent- } \\
\text { fernung der } \\
\text { Harnsäure. }\end{array}$ & $\begin{array}{l}\text { Nach Ent- } \\
\text { fernung der } \\
\text { Harnsäure. }\end{array}$ & \\
\hline $\begin{array}{l}1 \\
\left.2^{2}\right) \\
\left.3^{2}\right) \\
4 \\
5\end{array}$ & $\begin{array}{l}1,017 \\
1,020 \\
1,022 \\
1,023 \\
1,023\end{array}$ & $\begin{array}{lc}0,016 & \mathrm{pCt} . \\
0,06 \quad " \\
0,07 & " \\
0,07 & " \\
0,06 & "\end{array}$ & $\begin{array}{l}0,27 \mathrm{pCt} . \\
0,26 " \\
0,22 " \\
0,23 \quad " \\
0,33 "\end{array}$ & $\begin{array}{l}0,22 \mathrm{pCt} . \\
0,22 " \\
0,21 " \\
0,24 \quad " \\
0,25 \quad "\end{array}$ & $\begin{array}{l}0,21 \text { pCt. } \\
0,15 " ~ \\
0,17 " \\
0,18 " \\
0,29 "\end{array}$ \\
\hline
\end{tabular}

Nach diesen Versuchen kann kaum mehr als ein Viertel vom ursprünglichen Reductionsvermögen des Harns der Harnsäure zugeschrieben werden; zum grösseren Theil rührt dasselbe aller Wahrscheinlichkeit nach von nicht genauer bekannten Stoffen her; denn der verhältnissmässig unbedentende Kreatiningehalt des Harns kann nur in sehr untergeordneter Weise in Betracht kommen, da 1 Mol. Kreatinin nicht mehr als ea. 0,75 Mol. $\mathrm{CuSO}_{4}$ zu reduciren vermag. Auch bei der Entfärbung verloren die Harne bloss einen geringeren Antheil ihres ursprünglichen reducirenden Vermögens. Aus der Tabelle ergiebt sich, dass dieser Verlust nicht der Harnsäure allein zur Last fällt, denn in Nr. 2,

1) Der Procentgehalt der reducirenden Substanzen als Zucker berechnet.

2) Nr. 2 und 3 ergaben nach Kochung mit Wismuthoxyd in alkalischer Lösung Schwärzung; die übrigen nicht. 
3 und 4 reducirten die entfärbten Harne so viel weniger, als die ursprünglichen, aus welchen die Harnsäure ausgefällt war, dass man zu der Annahme berechtigt sein dürfte, dass die Kohle auch noch eine andere reducirende Substanz neben der Harnsäure aufnimmt. Kreatinin kann dies nicht sein, denn dieser Stoff wird nicht zurückgehalten, und dass die Spur ron Zucker, die in normalem Harn sich finden kann, nicht in Betracht kommt, ist durch specielle Gährungsversuche mit den Harnen Nr. 2 und 4 nachgewiesen, efr.

Nr. 2. Ursprünglicher Harn: Reductionsvermögen vor der Gährung 0,26 pCt., nach der Gährung 0,22 pCt. Entfärbter Harn: Redustionsvermögen vor der Gährung $0,15 \mathrm{pCt}$., nach der Gährung $0,14 \mathrm{pCt}$.

Nr. 4. Urspr. Harn: Reductionsvermögen vor der Gährung 0,23 pCt., nach der Gährung 0,22 pCt. Entfärbter Harn: Reductionsvermögen vor der Gährung 0,18 pCt., nach der Gährung 0,17 pCt.

Die reducirenden Substanzen, welche, ausser der Harnsäure, (wahrscheinlich) noch zurïckgehalten werden, sind also unbekannt, und ihre Menge, nach den Versuchen zu urtheilen, für die verschiedenen Harne in hohem Grade verschieden.

Die im Vorangegangenen mitgetheilten Resultate von Titrirungen mituelst der Knapp'schen Flüssigkeit lassen sich indessen kaum in ihrer ganzen Ausdehnang anf die Trommer'sche Probe überfübren; es dürfte vielmehr, aller Wahrseheinlichkeit nach, eine gewisse Abweichung sich geltend machen, da Harnsäure und Kreatinin viel leichter auf Kupferoxyd in alkalischer Mischung reducirend einwirken, als auf die Knapp'sche Flüssigkeit. Es würde daher von grösserer Bedeutung gewesen sein, wenn man vergleichende Bestimmungen mittelst der Fehling'sehen Flüssigkeit hätte ausführen können, aber weder der ursprüngliche, noch der entfärbte (zuckerfreie) Harn lassen sich nach dieser Methode direct titriren, da das Filtrat hier immer aufgelöstes Kupferoxyd enthält.

Unter allen Umständen darf man aus den mitgetheilten Versuchendas Recht zu dem Schluss ableiten, dass ein grösserer Bruchtheil des ursprïnglichen Reductionsvermögens nach der Entfärbung noch fortbesteht.

Auf Grund dessen, dass der entfärbte Harn immer noch 
Ueber das Verhalten des entfärbten Harns zu. Kupferoxyd a. Alkali etc. 131

reducirende und $\mathrm{k} u p f$ froxydulauflösende Substanzen einschliesst, darf man anch mit diesem beim Nachweis ron Zucker die Trommersche Probe nicht in der gewöhnlichen Weise anstellen. Auch hier muss man mit dem Zusatz von Kupfersulfat vorsichtig sein und die Erhitzung bis zum Kochpunkte vermeiden. Wendet man zu wenig Kupfersulfat an, so wird man blos Abfärbung erreichen, und setzt man zu viel zu, so wird man leicht blaugefärbte Flüssigkeit oder fein suspendirten Niederschlag von Kupferoxydhydrat erhalten, wodurch die Reaction sich maskirt. Die characteristische Fällung kann also ausbleiben, selbst wenn Zucker zugegen ist.

Macht man entfärbten Harn alkalisch und setzt Kupfersulfat zu, bis permanenter Niederschlag eintritt, so wird man bei Erhitzung auf $90-100^{\circ}$ schöne Ausscheidung von Kupferoxydulhydrat erzielen können, ohne dass der Harn Zucker zu enthalten braucht.

Es kann dies durch das Kreatinin bedingt sein, welches bei andanernder Erhitzung mit Alkali und Kupfersulfalt bis auf diese Temperaturen Fällung hervorruft. Nimmt man also bei entfärbtem Harne die Trommer'sche Probe in der gewöhnlichen Weise vor, so wird man auf dieselben Schwierigkeiten stossen, wie bei dem ursprünglichen.

Wie wir gesehen haben, gab die früher besprochene modificirte Trommer'sche Probe bei den ursprünglichen Harnen kein zuverlässiges Resultat auf Grund des Umstandes, dass unbekannte Stoffe Ausscheidung ron Kupferoxydulhydrat bewirkten. Man könnte sich nun die Möglichkeit davon denken, dass letztere durch die Kohle zurickgekalten würden und dass somit die Probe mit entfärbtem Harne sicherer ausfallen würde. Das Resultat entsprach indessen einer solchen Vermuthung nicht, insofern die zuckerfreien Harne, welche direct Ausfällung veranlassten, auch nach der Entfärbung Reaction ergaben, sowohl bei einer auf $60-70^{\circ} \mathrm{C}$. sich belaufenden Erwärmung mit $\mathrm{CuSO}_{4}$ und $\mathrm{KOH}$ im Wasserbade, als bei der modificirten Trommer'schen Probe. Es lag somit am Tage, dass jene Stoffe ihrem wesentlichen Antheil nach nicht von der Kohle zurïckgehalten werden, umsomehr als anch die Schwärzung von Wismuthoxyd in alkalischer Fitissigkeit ebenfalls nach der Entfärbung immer noch eintrat. Da die Reaction auch hier nach Hinstand mit Hefe nicht verschwand, so war hierdurch die Gegenwart von einer (resp. mehreren) bisher unbekannten reducirenden 
Substanzen in einer nicht geringen Anzahl normaler Harne nachgewiesen ${ }^{1}$, und es erscheint rationell vorzugsweise den entfärbten Harn zar näheren Untersuchung derselben anzuwenden.

\section{$\S 4$.}

\section{Ueber den Nachweis von Zucker im entfärbten Harne vermittelst Kupfersulfats und alkalischer Seignettesalzlösung.}

Wie früher mitgetheilt ${ }^{2}$, gaben ausnahmsweise einzelne (4) normale Harne beim Gebrauch der modificirten Fehlin g'schen Flüssigkeit, wenn dieselbe in der, in voriger Abhandlung angegebenen Weise angewandt wurde, Reaction, die durch Hefe nicht verschwand. Dies war auch der Fall mit dem entfärbten Harne, so dass die Probe durch die Entfärbung nicht an Sicherheit gewann. Wenn nun auch unter solchen Umständen der Entfärbung ke ine irgend wesentliche Bedeutung für den Nachweis von Zucker im Harne beigelegt werden konnte, so gewährte es doch Interesse eine exacte Vergleichung, in Bezug anf die Empfindlichkeit und Deutlichkeit der Reaction bei Anwendung von ursprïnglichem und entfärbtem Harn unter sonst gleichen Bedingungen anzustellen.

Gewöhnlicher zuckerfreier Harn wurde entfärbt, und darauf sowohl der entfärbte als der ursprüngliche mit einer bestimmten Menge Zucker versetzt, so dass man correspondirende Mischungen von $0,05,0,025,0,0167$ und 0,01 pCt. Zuckergehalt erhielt. Die Proben wurden in der friher angegebenen Weise ausgefuihrt. Es wurden $5 \mathrm{ccm}$ Harn, 2,5 cem Seignettesalzlösung und die passende Menge Kupfersulfatlösung $(2,5$ pCt.) angewendet.

Ursprünglicher Harn, mit Zusatz von 0,05 pCt. Zucker, gab Andeutung von Reaction bei $1,5 \mathrm{ccm} \mathrm{CuSO}_{4} ; 1 \mathrm{ccm}$ erwies sich als ungenügend und bei $2 \mathrm{ecm}$ blieb die Flüssigkeit blaugefärbt.

Ursprünglieher Harn, mit Zusatz von 0,025 pCt. Zucker, gab Reaction: ursprünglicher Harn mit Zusatz von $0,0167 \mathrm{pCt}$. Zucker dagegen keine, oder im besten Falle höchst undeutliche Reaction.

1) Man könnte vielleicht daran denken, dass das Kreatinin hier in Betracht käme; specielle Versuche mit ursprünglichen und entfärbten Harnen, die an und für sich keine Reaction gaben und denen $0,5 \mathrm{pCt}$. Kreatinin zugesetzt wurde, zeigten aber, dass dieses bei $70-75^{\circ} \mathrm{C}$. keinen derartigen Einfluss ausübte.

2) cfr. S. 112 , 
Ueber das Verhalten des entfärbten Harns zu Kupferoxyd u. Alkali etc. 133

Der entfärbte Harn, mit Zusatz von $0,05 \mathrm{pCt}$. Zncker, gab mit $0,6 \mathrm{~cm}$ Kupfersulfatlösung einige Minuten nach Mischung der erwärmten Flüssigkeiten charakteristisch gelbe Fällung; $0,4 \mathrm{ccm}$ erwies sich hier als zu wenig, und bei $0,8 \mathrm{~cm}$ hielt die Flüssigkeit sich blaugefärbt.

Entfärbter Harn von 0,025 pCt. Zuckergehalt gab mit $0,3 \mathrm{ccm}$. Kupfersulfatlösung nach 4-5 Minuten schöne Reaction; $0,2 \mathrm{ccm}$ war zu wenig, und bei $0,5 \mathrm{ccm}$ war die Flüssigkeit schwach bläulich.

Entfärbter Harn, mit Zusatz von 0,0167 pCt. Zucker gab mit 0,3 ccm Kupfersulfatlösung Ausscheidung von feinem suspendirten Kupferoxydulbydrat.

Entfärbter Harn, mit Zusatz von 0,01 pCt. gab dagegen ein negatives Resultat.

Die Probe schien hier bei dem entfärbten Harn sich ein wenig feiner anzulassen, aber man muss sich hüten, aus einer einzelnen Versuchsreihe allgemeine Schlüsse ziehen zu wollen, da das Resultat bei anderen Versuchen eher'das entgegengesetzte war efr.

Ursprünglicher Harn von 0,05 pCt. Zuckergehalt gab schöne Reaction nach 2 Minuten mit $2 \mathrm{ccm} \mathrm{CuSO}_{4} ; 1,5 \mathrm{~cm}$ war zu wenig und bei $2,5 \mathrm{ccm}$ hielt die Flüssigkeit sich bläulich. Derselbe Harn mit 0,025 pCt. Zuckergehalt gab deutliche Reaction nach 5 Ninuten mit $2 \mathrm{ccm} \mathrm{CuSO}_{4}$.

Derselbe Harn von 0,0167 pCt. Zuckergehalt gab Andeutung von Reaction bei $2 \mathrm{ccm} \mathrm{CuSO}_{4}$.

Es wurde der Harn entfärbt und demselben Zucker zugesetzt.

Entfärbter Harn von 0,05 pCt. Zuckergehalt gab schöne Reaction bei $1,75 \mathrm{ccm} \mathrm{CuSO}_{4} ; 1,5 \mathrm{ccm}$ war auch hier zu wenig.

Derselbe Harn von 0,025 pCt. Zuckergehalt gab deutliche Reaction nach 5 Minuten mit $2 \mathrm{ccm} \mathrm{CuSO}_{4}$; Reaction traf auch noch ein bei Anwendung von 1,75 cem $\mathrm{CuSO}_{4}$, während $1,5 \mathrm{ccm}$ sich auch hier als ungenügend erwies.

Derselbe Harn von 0,0167 pCt. Zuckergehalt gab negatives Resultat.

Diesmal waren also die Proben beim ursprünglichen Harn eher feiner, insofern es hier gelang $0,0167 \mathrm{pCt}$. nachzuweisen, während man in dem entfärbten Harn nur bei 0,025 pCt. Reaction erhielt.

In diesem Fall schien also das reducirende Vermögen des Harns durch die Entfärbung in höherem Grade herabgedrückt zu sein, als das Vermögen zur Lösung des Kupferoxyduls, während in der vorangehenden Versuchsreibe das entgegengesetzte stattfand. Wodurch dieser (geringfigige) Wechsel im Verhalten bedingt wird, wage ich nicht mit Bestimmtheit auszusprechen. 
Die Probe gewann also durch die Entfärbung kaum an Empindlichkeit, und jedenfalls war dieselbe bei Weitem nicht so fe in, wie in einer wässrigen Zuckerlösung. Bei letzterer glïckte es vermittelst der angewendeten Methode constant bei einem Zuckergehalt von 0,0075 pCt. Reaction zu erhalten, und in der Regel sogar bei einem Zuckergehalt von $0,005 \mathrm{pCt}$. in $5 \mathrm{ccm}$. Mit andern Worten: auch der entfärbte Harn enthält einen Ueberschuss an kupferoxydullösender Substanz.

Die Fällung trat indessen in dem entfärbten Harn so deutlich und so rein zu Tage, dass man meinen könnte, letzterem sei aus diesem Grunde jedenfalls der Vorzug zu geben, wenn auch die Reaction an und für sich nicht empfindlicher ist.

Aber abgesehen davon, dass es bequemer ist, die Reaction mit dem ursprünglichęn Harn anzustellen, darf man es nicht als selbstverständlich ansehen, dass der entfärbte Harn denselben Zuckergehalt besitzt, wie der ursprüngliche, da die Kohle eine gewisse Zuckermenge zurïckhalten kann. Man darf nicht ohne Weiteres die hier gemachten Erfahrungen in Betreff der Empfindlichkeit der Probe auf zuckerhaltigen Harn, der durch Kohle entfärbt ist, übertragen.

Es zeigte sich auch in der That bei vergleichenden Versuchen, dass ein wenig Zucker von der Kohle zurïckgehalten wurde, da die Reaction etwas empfindlicher zu sein schien, wenn dem entfärbten Harn direct 0,025 pCt. Zucker zugesetzt wurde, als wenn man den ursprünglichen Harn von gleichem Zuckergehalt durch Kohle filtrirte, efr. folgenden Versuch:

Entfärbter Harn von 0,025 pCt. Zuckergehalt gab deutliche Reaction. Dagegen war nach der Filtration des ursprünglichen Harns von $0,025 \mathrm{pCt}$. durch Knochenkohle die Reaction, welche erst nach 5 Minuten eintrat, verhältnissmässig schwach, wenn auch deutlich.

Obwohl der Unterschied gering ist, so dass die so zurückgehaltene Zuckermenge die Empfindlichkeit kaum in merkbarer Weise beeinträchtigt, schien es doch immerhin für die practische Beurtheilung nöthig, zu untersuchen, wie die Probe ausfällt, wenn zuckerhaltiger Harn dureh Kohle filtrirt wird; za dem Ende wurde eine grössere Anzahl Harne von 0,05-0,025 $-0,0167 p \mathrm{Ct}$. Zuckergehalt durch Kohle filtrirt und die Reaction vor und nach der Entfärbung verglichen. Ganz so wie in den vorangehenden Versuchen wurde auch hier die Empfindlichkeit der 
Ueber das Verhalten des entfärbten Harns zu Kupferoxyd u. Alkali etc. 135

Probe in der Regel weder verschärft, noch gesehwächt durch die Entfärbung ${ }^{1}$ ).

Wenn aber somit die Reaction aurch die Filtration durch Kohle nicht eigentlich an Empfindlichkeit gewinnt, ist dennoch die Probe am entfärbten Harn ein unschätzbares Controllmittel, weil die Kupferoxydulbydratfällung bei letzterem immer reiner gelb ist und desshalb nicht zu Verwechselungen Anlass geben kann, wäbrend solche, wie früher nachgewiesen, bei dem ursprünglichen Harne vielleicht jezuweilen eintreten können, wenn der Zuckergehalt gering ist.

$\S 5$.

Ueber die knpferoxydallösenden Substanzen des Harns.

Die Harnsäure kann, wie früher gezeigt, eine gewisse Menge $\mathrm{Cu}_{2} \mathrm{O}$ gelöst halten, wird aber so viel $\mathrm{CuSO}_{4}$ zugesetzt, als die Harnsäure reduciren kann, so wird das durch die Oxydation des Zuekers gebildete Kupferoxydul zum Vorsehein kommen, und die Probe im Wesentlichen ebenso empfindlich sein, wie in wässriger Zuckerauflösung. Mit diesem Umstand vor Augen habe ich den Kupfersulfatzusatz methodiseh variirt und gefunden, dass bei einer passenden Menge $\mathrm{CuSO}_{4}$ in zuckerhaltigem Harne constant typische Ausseheidung eintritt, aber nur bis zu einer bestimmten Grenze: nämlich ca. $0,02-0,025$ pCt. Da diese Grenze im Wesentlichen dieselbe ist, ob die von mir modificirte Probe im ursprünglichen oder im entfärbten Harn vorgenommen wird, so leuchtet ein, dass diejenige Substanz, welche die Ausfällung von $\mathrm{Cu}_{2} \mathrm{O}$ bei einem Zuckergehalt von unter 0,025 pCt. verhinderte und deren Einfluss sich nicht eliminiren liess, von der Kohle nicht zurückgehalten wurde, und kommt dann das Kreatinin zunächst in Betracht. Ich habe früher nachgewiesen, wie man durch Bezugnahme auf letzteren Stoff sich darïber Rechenschaft geben kann, warum bei einer kleinen Menge $\mathrm{CuSO}_{4}$ kein Niederschlag auftritt, und warum man, auch wenn man stufenweise den $\mathrm{CuSO}_{4}$-gehalt steigert, minimale Zuckerquantitäten nicht wird nachweisen können, da bereits

1) Ganz dasselbe Resultat ergab sich bei der Untersuchung von diabetischen Harnen, welche bloss eine Spur von Zucker erhielten. 
ein Kreatiningehalt von ea. 0,09 pCt. den Nachweis von $0,02 \mathrm{pCt}$. Traubenzucker fast unmöglich macht. Auf demselben Weg wird man sich auch erklären können, wesshalb die Empfindlichkeit für die verschiedenen Harne verschieden ist. Während in vielen von diesen erst bei 0,025 pCt. Zuckergehalt Ausscheidung eintritt, trifft es sich, dass man in andern bei 0,02 pCt., ja sogar bis 0,0125 pCt. Fällung erzielt, wie dies durch eine Reihe von Versuchen mit ausgegohrenen Harnen, denen man $\left.{ }^{1}\right)$ kleine Quantitäten Zucker zugesetzt hatte, zur Genüge bewiesen worden. Dies lässt sich leicht verstehen, wenn man in Betracht zieht, dass der Kreatiningehalt des Harns wechseln kann (in Neubauer's Bestimmungen von $0,05-0,08$ pCt.), denn wir baben gesehen, dass 0,07 pCt. Kreatinin der Nachweisung von 0,02 pCt. Zacker keine wesentlichen Hindernisse in den Weg legt, und dass man bei 0,05 pCt. Kreatinin sogar bis $0,0015 \mathrm{pCt}$. Zucker nachweisen konnte.

Mit Bezug darauf, dass $\mathrm{NH}_{3}$ Kupferoxydul aufgelöst halten kann, lag es nahe, anzunehmen, dass Harnstoff, wenn auch in geringerem Grade, einen ähnlichen hemmenden Einfluss auf die Empfindlichkeit der Probe ausiiben könnte. Um hierüber Auskunft zu erbalten, wurde eine Reihe von Versuchen mit 2-3-4. pCt. Harnstoffauflösungen von geringem Zuckergehalt angestellt, aber die Reaction zeigte sich hier ebenso fein, wie in wässerigen Zuckerauflösungen von geringem Zuckergehalt, so dass es - jedenfalls im entfärbten Harne - aller Wahrscheinlichkeit nach nur das Kreatinin ist, welches den störenden Einfluss ausïbt.

$\$ 6$.

\section{Ueber die Probe im Waschwasser.}

Die Probe am entfärbten Harn giebt nach dem Gesagten somit an und für sich kein sichreres Kennzeichen für Zucker, als diejenige am urspriinglichen. Die Reaction im entfärbten Harn kann darum nur secundär in Betracht kommen; denn der grosse Vortheil, die Probe direct am ursprünglichen Harn vornehmen zu können, ist ohne weiteres einleuchtend.

1) nachdem sie zuerst zum Kochen erhitzt waren, um weitere Gährung zu verbindern. 
Ueber das Verhalten des entfärbten Harns zu Kupferoxyd u. Alkali etc. 137

Wenn Seegen ${ }^{1}$ ) und Maly ${ }^{2}$ ) angeben, dass die Trommer'sche Probe im entfärbten Harn viel empfindlicher sein soll, so kann diese Behauptung nur darauf beruhen, dass sie unter anderen Bedingungen operirt haben. Aber auf der anderen Seite muss man einräumen, dass die Ausfällung von Kupferoxydul im entfärbten Harne charakteristischer ist, als in dem ursprünglichen. In letzterem tritt nämlich bei einem Zuckergehalt von ca. $0,1 \mathrm{pCt}$ nur Ausscheidung von gelbem Kupferoxydulhydrat auf, während man beim entfärbten Harn bei diesen Concentrationen häufig schönen rothen Niederschlag von $\mathrm{Cu}_{2} \mathrm{O}$ erbält. Das kupferoxydullösende Vermögen machte sich dagegen auch in dem entfärbten Harne so stark geltend, dass die Reaction bei einem Zuckergehalt ron weniger als $0,025 \mathrm{pCt}$. inconstant und unzuverlässig wurde.

Seegen, der sich davon ibberzengt hat, dass der entfärbte Harn immer noch kupferoxydullösende Substanzen enthält, und der nachgewiesen hat, dass die Kohle eine gewisse Menge Zucker zurïckhält, insofern der Procentgehalt der filtrirten Fliussigkeit an diesem Stoff constant geringer ist, als der des ursprünglichen, hat auf dieser Grundlage den Vorschlag gemacht, die Kohle nach der Filtration mit destillirtem Wasser auszuwaschen und die Probe im Waschwasser anzustellen, und es soll nach ihm die Reaction in dieser Weise bedeutend an Empfindlichkeit gewinnen; efr.: "Die Reduction wird dagegen eine sehr charakteristische, wenn nach vollendeter Filtration die auf dem Filter befindliche Kohle mit wenig destillirtem Wasser gewaschen und das Waschwasser zur Probebenutzt wird". Namentlich soll die Probe, nach seiner Angabe, an Feinheit gewinnen bei Anwendung des 2. und 3. Waschwassers, efr.: „Ich beobachtete ferner, dass in diesen Fällen das zweite und dritte Waschwasser eine viel charakteristischere Reaction gab als das erste. Während das erste Waschwasser eine noch etwas missfürbige Trübung mit der Fehling'schen Lösung hervorbrachte, gaben die folgenden Waschwässer eine schöne Ausscheidung von gelbem Kupferoxydulhydrat, und selbst das vierte Waschwasser zeigte eine zwar nur minimale Zuckermenge

1) Seegen, dies Archiv, Bd. V, 1872, S. 375-378.

2) Maly, Sitzungsberichte d. k. Akad. dex Wissenschaften, math.-naturw. Classe, Wien 1871, Bd. 63, S. 484. 
an, aber es geschah in derselben charakteristischen Weise, wie das in einer wässerigen Zuckerlösung mit minimalem Zuckergehalte geschieht" ${ }^{1}$ ).

Dass das zweite und dritte Waschwasser weniger kupferoxydullösende Substanz entbält, ist leicht zu verstehen, da eine gewisse Menge Harn nach der Filtration noch in der Kohle zugegen ist, aber durch die erste Auswaschung wesentlich entfernt wird, wesshalb das erste Waschwasser mehr von demselben enthalten wird, als das 2. und 3. Indessen ist die Zuckermenge, welche durch die 2. und 3. Auswaschung entfernt 'wird, da, wo es sich nur um Spuren handelt, so gering, dass die Probe, in der angegebenen Weise vorgenommen, für jede Auswaschung an Empfindlichkeit verliert. Die Reaction ist darum feiner sowohl in dem ursprïnglichen und dem entfärbten Harn, als im Waschwasser.

Seegen hat nicht genauer mitgetheilt, was er unter dem 2. und 3. Waschwasser versteht, insofern er nicht das Quantum von Flüssigkeit angiebt, welches bei jeder Auswaschung angewendet wurde, und darum ist es auch nicht ganz leicht, direct seine Angaben zu controlliren. Meine Versuche wurden in der Weise ausgefthiht, dass ich nach der Filtration durch Kohle diese mit einem Quantum Wasser auswässerte, welches dem Quantum des Filtrats entsprach. Das so erhaltene Filtrat bezeichne ich als erstes Waschwasser. Wird die Auswaschung (in derselben Weise) wiederholt, erhält man das zweite Waschwasser u. s. w. Es bat sich nun herausgestellt, dass die Reaction im Waschwasser bei ursprünglich geringem Zuckergehalt sich nicht in der hier angegebenen Weise anstellen lässt, sondern, dass man bis zum Kochen erhitzen muss. Aber trotz alles Kochens gelang es mir, bei einem ursprünglichen Zuckergehalt von 0,1 pCt, doch nicht im zweiten und dritten Waschwasser deutliche Reaction zu erhalten; im ersten Waschwasser war die Reaction bei $0,1 \mathrm{pCt}$. freilich sehr gut; dagegen lässt sich $0,05 \mathrm{pCt}$. nur ausnahmsweise auf diesem Wege nachweisen.

Ich habe die Versuche anch in der Weise modificirt, dass ich bei jeder Auswaschung eine kleinere Wassermenge benutzt habe (ungefähr die Hälíte des Filtrats), aber das Resultat war auch hier nur wenig befriedigend.

1) Seegen, 1. c. S. 376 und 377 . 
Ueber das Verhalten des entfärbten Harns zu Kupferoxyd u. Alkali etc. 139

Diese Methode zur Anstellung der Probe ist also nach meiner Erfahrung nicht anzurathen. Freilich enthält das zweite und dritte Waschwasser nur eine verhältnissmässig geringe Menge kupferoxydullösender Substanz, aber das Zuckerquantum, welches durch die Auswaschung entfernt wird, ist unter diesen Umständen so geringfiigig und möglicherweise auch so variabel, dass die in solcher Art angestellte Probe als unsicher angesehen werden muss.

\section{Berichtigungen.}

S. 48 , Z. 17 von u. statt: 0,0016 pCt. Ur lies: 0,016 pCt. Ur.

S. 49, Z. 1 von u. statt: $0,009 \mathrm{ccm}$ lies: $0,09 \mathrm{ccm}$.

S. 53, Z. 9 von 0 . statt: Kupfer reducirt lies: Kupferoxyd reducirt.

S. 55, Z. 11-12 von u. statt: wurden $0,6 \mathrm{ccm}$ lies: wurden mit $0,6 \mathrm{ccm}$.

S. 62, Z. 9-10 von o. statt: $\mathrm{CuO}$ mit voller Sicherheit reducirt lies: $\mathrm{CuO}$ reducirt.

S. 63, Z. 2-3 von o. statt: Bei 0,77 und 0,8 Mol. Kreatinin lies: Bei 0,77 und 0,8 Mol. $\mathrm{CuSO}_{4}$ auf 1 Mol. Kreatinin.

S. $63, \mathrm{Z} .9$ von o. statt: $\mathrm{CuSO}+5 \mathrm{HO}_{2}$ lies: $\mathrm{CuSO}_{4}+5 \mathrm{H}_{2} \mathrm{O}$.

S. 66 , Z. 19 von u. statt: von gelbgrünem $\mathrm{Cu}(\mathrm{OH})_{2}$ lies: von gelbgrünem $\mathrm{Cu}_{2}(\mathrm{OH})_{2}$.

S. 70 , Z. 2 von u. statt: Jahrgang 1875 lies: Jahrgang 1871.

S. 78 , Z. 8 von o. statt: folgenden Tabellen lies: der folgenden Tabelle.

S. 82 , Z. 14 von u. statt: $\mathrm{Cu}_{2}(\mathrm{OH})_{2}$ lies: $\mathrm{Cu}(\mathrm{OH})_{2}$.

S. 88, Z. 5 von o. statt: im letzteren Falle lies: im ersteren Falle.

S. 104, Z. 2 von 0. statt: deutliche Ausfällung lies: keine deutliche Ausfällung.

S. 106, Z. 15 von u. statt: suspendirten Kupferoxydulverbindungen lies: suspendirten Kupferoxydverbindungen. 